\title{
A STUDY ON GAIN SCHEDULING OF INTELLIGENT EXCAVATOR
}

\author{
Chang-Seop Lee, Dongnam Kim, \\ Korea University Graduate School, Seoul, Korea \\ acelcs@hanmail.net \\ Baeksuk Chu, Daehie Hong \\ Korea University, Seoul, Korea \\ dhhong@korea.ac.kr
}

\begin{abstract}
One of the most important factors in Intelligent Excavator is to locate the bucket quickly and accurately to the right position. To locate the bucket accurately, the Arm and the hydraulic cylinder of the boom should be exactly controlled. But as voltage and the force that should be controlled can be different by the positioning of the excavator, exact controlling is a difficult task. For more accurate control of position of bucket tip, we are going to apply gain scheduling algorithm. In this paper, we looked on how much the excavator could be controlled with gain scheduling algorithm.
\end{abstract}

\section{KEYWORDS: Control, Gain Scheduling, Position Control, Intelligent Excavator}

\section{INTRODUCTION}

From remote control excavator to unmanned robotic excavator, researches on automation of excavator are conducted continuously worldwide. The excavator has been trying to be intelligent, while the conventional excavator with the problems kind of the efficiency, the safety. The most important thing of intelligent excavator system is to move the bucket to the desired point quickly and accurately. For controlling the position of bucket accurately, the displacement of hydraulic cylinders on the arm and the boom has to be controlled accurately. However, it is not easy to control the hydraulic cylinder, because the force and control input voltage is different according to the environment and the pose of the excavator. For more accurate control of position of bucket tip, we are going to apply gain scheduling algorithm. In this paper, we looked on how much the excavator could be controlled with gain scheduling algorithm.

\section{EXCAVATOR KINEMATICS}

\section{Forward Kinematics}

The kinematics of the robot was derived using D-H convention from Denavit and Hartenberg (Paul, 1981). Because the manipulator lacks swing motion of excavator it can be seen as planer manipulator. Planer manipulator has 2 DOFs for end-effector position, but excavator has 3 planer links, therefore redundancy occurs. To handle the redundancy issue, user must specify bucket angle, which is measured from reference frame. Therefore, once bucket angle is defined, with trigonometric relationships inverse kinematics solution can be 
derived without any problem. There are for homogenous transformation matrices and calculation does not cost much. Coordinate transformations for the manipulator will be derived as follows:

$$
{ }^{0} T_{4}={ }^{0} T_{1}^{1} T_{2}{ }^{2} T_{3}^{3} T_{4}
$$

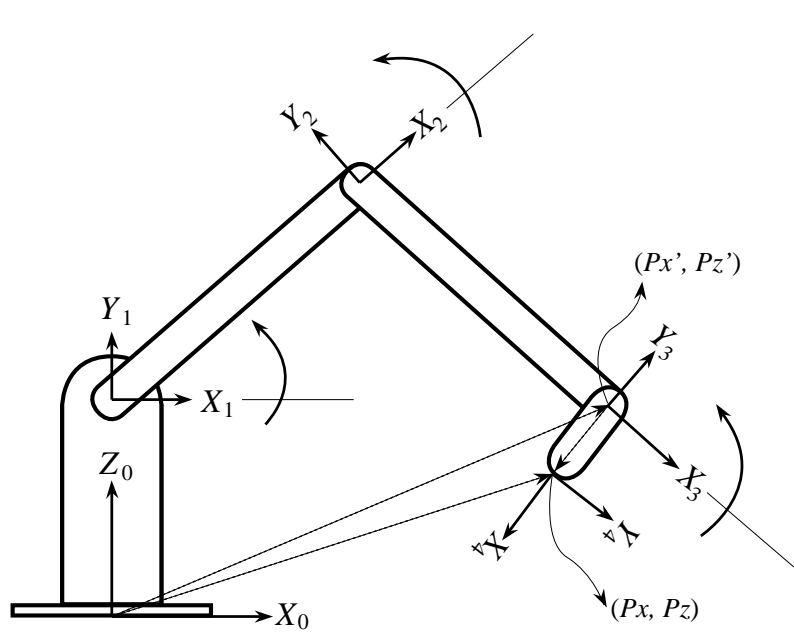

Figure 1: Excavator joint variables

In the same way, the hand position from reference frame can be described as:

$$
{ }^{R} T_{H}=\left[\begin{array}{cccc}
\cos \theta_{y} & 0 & -\sin \theta_{y} & p_{x} \\
0 & 1 & 0 & 0 \\
\sin \theta_{y} & 0 & \cos \theta_{y} & p_{y} \\
0 & 0 & 0 &
\end{array}\right]
$$

$\theta_{y}$ is the angle of bucket and $p_{x}, p_{y}$ is the end-effector position relative to reference frame which can be obtained as:

$$
\begin{aligned}
& p_{x}=a_{2} \cos \theta_{2}+a_{3} \cos \theta_{23}+a_{4} \cos \theta_{234} \\
& p_{y}=a_{2} \sin \theta_{2}+a_{3} \sin \theta_{23}+a_{4} \sin \theta_{234}+d_{1}
\end{aligned}
$$

\section{Inverse Kinematics}

As can be seen in the Figure 1 , end-effector position $\left(p_{x}, p_{y}\right)$ and $\theta_{y}$ is given. From the configuration, values of $p_{x}{ }^{\prime}$ and $p_{y}^{\prime}$ can be obtained from following relationship.

$$
\begin{aligned}
& p_{x}^{\prime}=p_{x}-a_{4} \cos \theta_{y} \\
& p_{y}^{\prime}=p_{y}-d_{1}-a_{4} \sin \theta_{y}
\end{aligned}
$$

Other angular values can be derived from trigonometric rules such that: 


$$
\begin{gathered}
\theta_{3}=\operatorname{atan} 2\left(\frac{a_{2}^{2}+a_{3}^{2}-\left(p_{x}^{\prime 2}+p_{y}^{\prime 2}\right)}{2 a_{2} a_{3}} \sqrt{1-\left\{\frac{a_{2}^{2}+a_{3}^{2}-\left(p_{x}^{\prime 2}+p_{y}^{\prime 2}\right)}{2 a_{2} a_{3}}\right\}^{2}}-\pi\right. \\
\theta_{2}=\left|\begin{array}{ll}
a_{2}+a_{3} \cos \theta_{3} & -a_{3} \sin \theta_{3} \\
a_{3} \sin \theta_{3} & a_{2}+a_{3} \cos \theta_{3}
\end{array}\right|^{-1}\left|p_{x}^{\prime}\right| p_{y}^{\prime} \mid \\
\theta_{4}=\theta_{y}-\theta_{3}-\theta_{2}
\end{gathered}
$$

\section{EXCAVATOR DYNAMICS}

\section{Force of Cylinder}

We have to consider about the friction in the inner side of cylinder in order to calculate the force generated at each cylinder. The friction of the cylinder rod between working fluid in the hydraulic cylinder is non linear. Because of the friction is affected by not only the length of cylinder but also the pressure, it is not easy to know accurately the friction in the cylinder. There were some previous studies about calculating the friction in cylinder, viscous-coulomb model, Bonchis model, non linear system identification using Hammerstein model and so on. Equation (8) shows the calculation of the force generated at the cylinder. The equation of friction is equation (9) applied by Bonchis Model. We make the each links of excavator be uniform velocity and get the relation equation to calculate the friction from equation (9), (10).

$$
\begin{gathered}
F_{\text {hydr }}=A_{1} p_{1}-A_{2} p_{2} \\
M \ddot{x}=F_{\text {hydr }}-F_{\text {friction }} \\
F_{\text {friction }}=x_{1} e^{x_{2} v}+x_{3}\left(p_{1}-p_{2}\right)+x_{4} p_{2}+x_{5} v
\end{gathered}
$$

$A_{1}, A_{2}$ is respectively the area of the piston head and rod, $p_{1}, p_{2}$ is the pressure of inner side of cylinder, $x_{1}, x_{2}, \ldots, x_{5}$ is coefficients to be determined and $v$ is cylinder length velocity.

\section{Torque of Joint}

Next step, we have to transform the force of cylinder (8) to the torque of each joint. Figure 2 shows the analysis of kinematics of Boom by change of cylinder length. Eq. (11) (15) is the relation between the force generated at cylinder and the torque by change of cylinder length.

$$
\theta_{1 B A}=\cos ^{-1}\left(\frac{l_{1}^{2}+r_{1 B}^{2}-r_{1 A}^{2}}{2 l_{1} r_{1 B}}\right)
$$




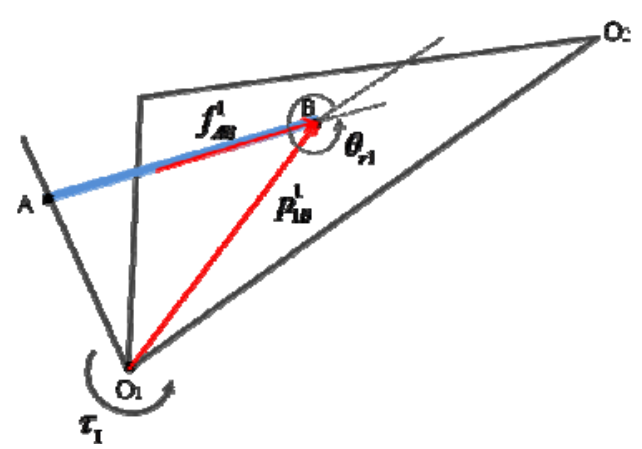

Figure 2: Kinematics of Boom by change of cylinder length

$$
\begin{gathered}
\theta_{r 1}=2 \pi+\left(\theta_{B 12}-\theta_{1 B A}\right) \\
\theta_{r 1}=2 \pi+\left(\theta_{B 12}-\theta_{1 B A}\right) \\
f_{A B}^{1}=|f(1)|\left[\begin{array}{lll}
\cos \left(\theta_{r 1}\right) & \sin \left(\theta_{r 1)} \quad 0\right.
\end{array}\right]^{T} \\
\tau_{1}=\left(p_{1 B}^{1} \times f_{A B}^{1}\right) \cdot z_{1}
\end{gathered}
$$

$l_{1}$ is the length of boom cylinder, $r_{1 B}$ is the distance between $O_{1}$ and $\mathrm{B}, p_{1 B}^{1}$ is the mass center position vector of $\mathrm{B}$ and $O_{1}, f_{A B}^{1}$ is the force vector generated at cylinder.

\section{Excavator Lagrangian Dynamics Model}

The force at end of the bucket can be guessed by using the Lagrangian dynamic model and the relation equation of torque. Lagrangian dynamic model is consist of the terms kinetic energy and potential energy (16). Eq. (17) and Eq. (18) is about kinetic energy and potential energy.

$$
\begin{gathered}
L=K-U \\
K=\frac{1}{2} \sum_{i=1}^{n} v_{c i}^{T} m_{i} v_{c i}+\omega_{i}^{T} I_{i} \omega_{i} \\
U=-\sum_{i=1}^{n} m_{i} g^{T} p_{o, c i}
\end{gathered}
$$

$v_{c i}=$ trnaslational velocity vector

$\omega_{i}=$ rotational velocity vector

$m=$ mass matrix

$I_{i}=$ inertia matirx

$g=$ gravitational vector

$p_{o, c i}=$ position vector of the center of masses 
Differentiate and rearrange the Eq. (16), (17), (18), we can get the Eq. (19), Eq. (20) about the torque.

$$
\begin{gathered}
\sum_{j=1}^{n} m_{i j} \ddot{\theta}_{j}+\sum_{j=1}^{n} \sum_{k=1}^{n}\left(\frac{\partial M_{i j}}{\partial \theta_{k}}-\frac{1}{2} \frac{\partial M_{j k}}{\partial \theta_{i}}\right) \dot{\theta}_{j} \dot{\theta}_{k} \\
-\sum_{j=1}^{n} m_{j} g^{T} J_{v j}^{i}=\tau_{i} \\
M(\theta)(\ddot{\theta})+V(\theta, \dot{\theta})+G(\theta)=\tau
\end{gathered}
$$

\section{SYSTEM OF EXCAVATOR}

The intelligent excavator consists of the controller, sensors and PXI on the 14 ton conventional excavator. The driver can control the whole system of excavator, using the computer which can communicate with the PXI system. The system has also the wire encoders on the each link which can measure the position and the angle of each hydraulic cylinder at Figure 4. Figure 3 shows the whole system and the flow of communication.

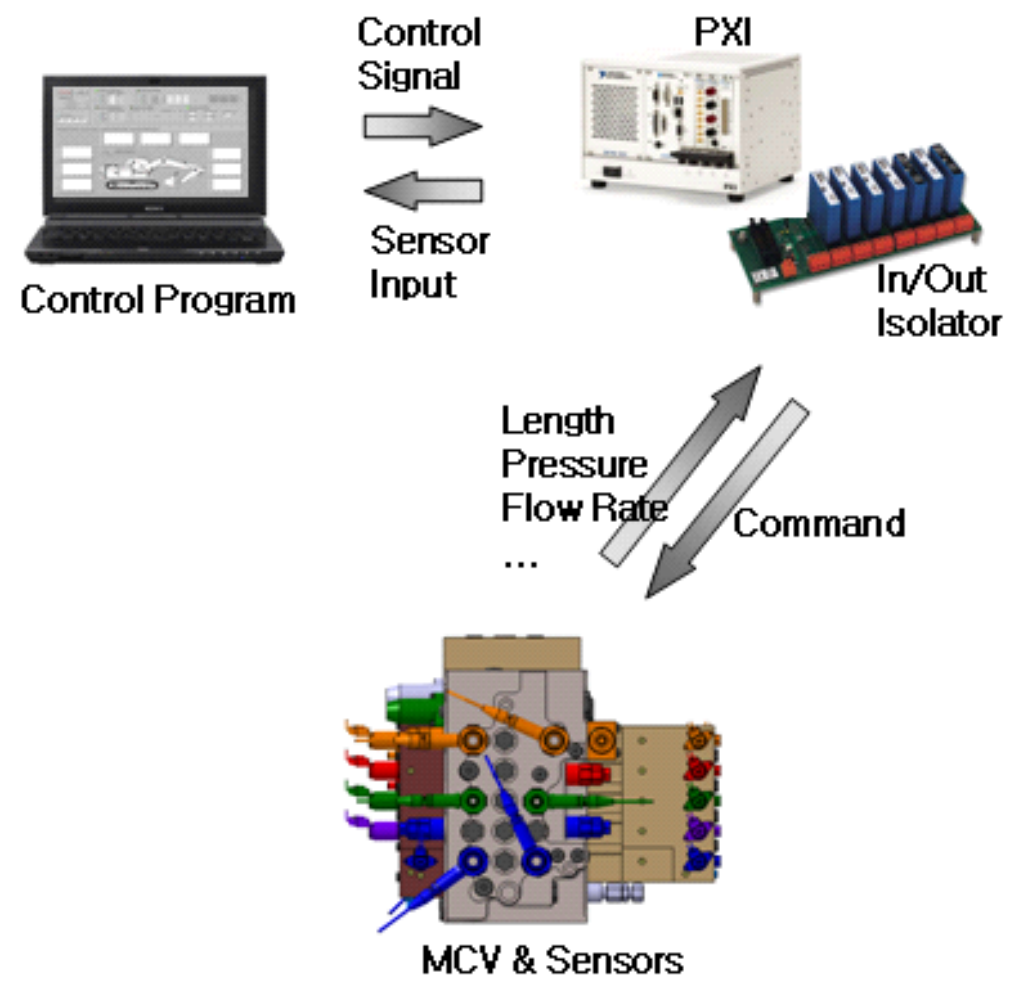

Figure 3: Schematic diagram of control signal 


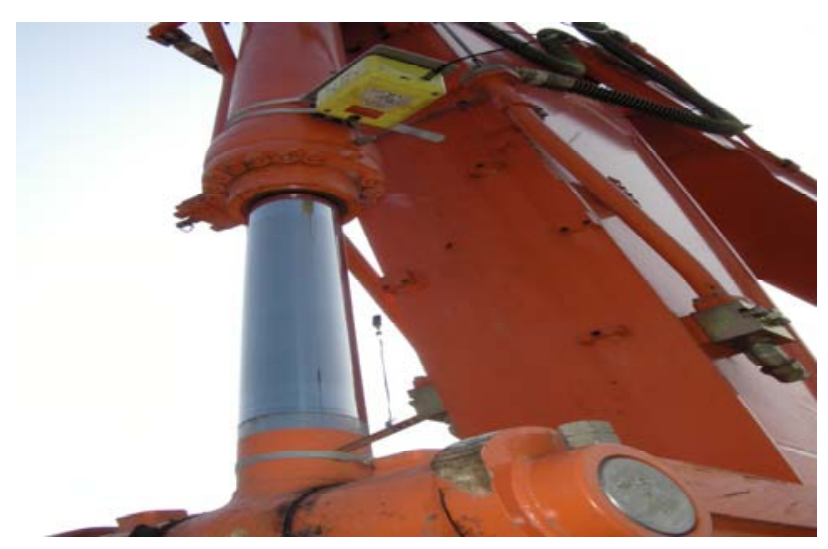

Figure 4: Wire encoder on bucket cylinder

The intelligent excavator system is programmed using the LabView 8.5 and the whole algorithm is like figure 5. Basically this system has PI controller and plus gravity compensation and gain scheduling. Gravity compensation and gain scheduling is applied by feed forward.

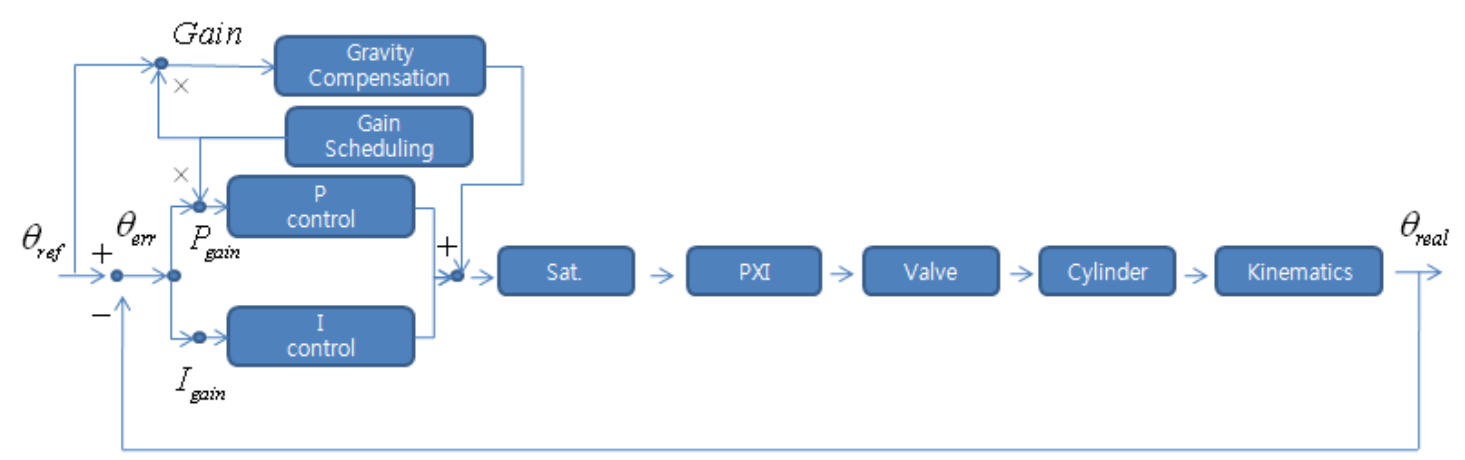

Figure 5: Block diagram of system

\section{GAIN SCHEDULING AND GRAVITY COMPENSATION}

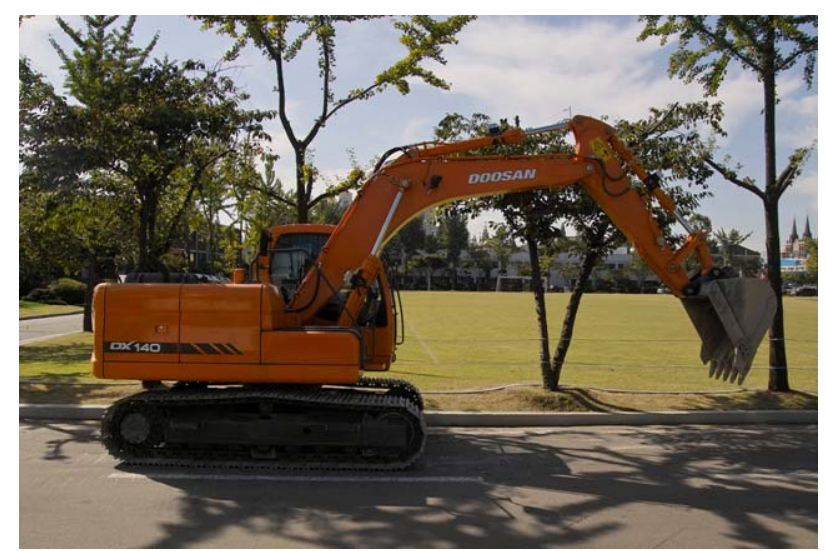

Figure 6: Intelligent excavator 
The important thing of excavator controller is the pose and gravity's effect. Like the figure 6, if the bucket is far from the base, the excavator needs more force. On the other hand, the tip of bucket is close to the base, it needs less force respectively. In actual field test, the control input voltage is different according to the pose of excavator. The below equation is about additional control input voltage which the excavator needs according to the pose of excavator. This additional control input makes the performance of excavator more flexible.

$$
\begin{aligned}
G_{2}= & M_{3} \times g \times\left(L_{\mathrm{O}_{1} \mathrm{O}_{2}} \times \cos \theta_{1}+L_{\mathrm{O}_{2} \mathrm{O}_{3}} \times \cos \left(\theta_{1}+\theta_{2}\right)+L_{\mathrm{O}_{3} \mathrm{G}_{4}} \times \cos \left(\theta_{1}+\theta_{2}+\theta_{3}+\theta_{3 m c}\right)\right) \\
& +M_{2} \times g \times\left(L_{\mathrm{O}_{1} \mathrm{O}_{2}} \times \cos \theta_{1}+L_{\mathrm{O}_{2} \mathrm{G}_{3}} \times \cos \left(\theta_{1}+\theta_{2}+\theta_{2 m c}\right)+M_{1} \times g \times\left(L_{\mathrm{O}_{1} \mathrm{G}_{2}} \times \cos \left(\theta_{1}+\theta_{1 m c}\right)\right.\right.
\end{aligned}
$$

Furthermore, when the hydraulic cylinder is moving forward and then reward, or when the movement of hydraulic cylinder is faster, there are the vibration and poor performance on the excavator because of the friction of inner cylinder. In this case, the controller has to be compensated more gain value for overcoming the friction of inner cylinder. Figure 7 shows the result of experiement without gain scheduling and gravity compensation. When the bucket reaches maximum position and then controller tries to move up the boom, there are much vibration and error of position.

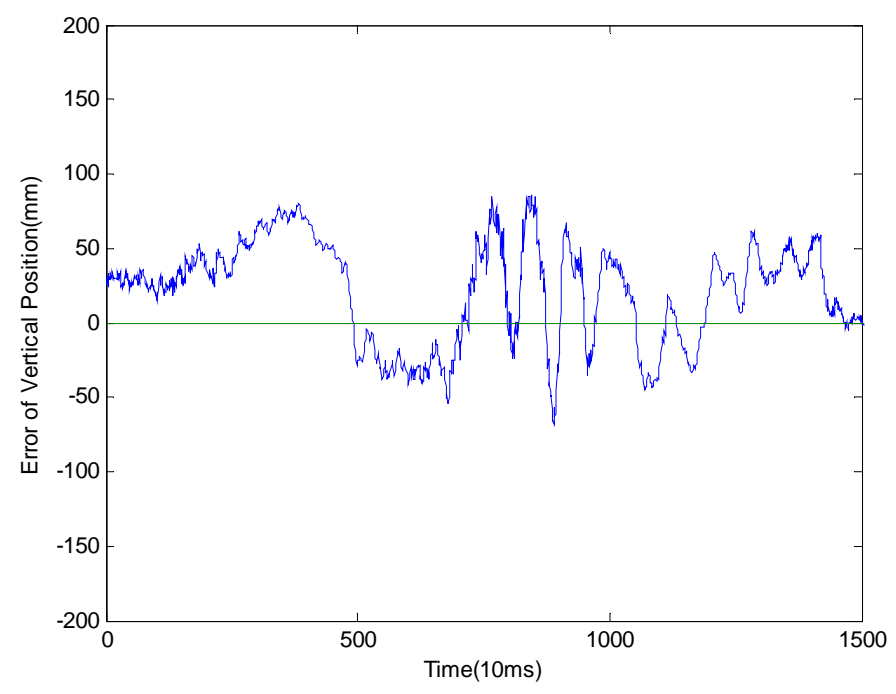

Figure 7: Error of vertical bucket's position without gravity compensation and gain scheduling

\section{EXPERIMENT AND RESULT}

In the field test, the tip of bucket is on the line of land and the tip moves $2.5 \mathrm{~m}$ forward and then reward to the first position. The velocity is $0.5 \mathrm{~m} / \mathrm{sec}$ and the sampling time is $10 \mathrm{msec}$. Figure 8 shows the error of bucket's vertical position with gravity compensation and gain scheduling. As the overall error is between $\pm 5 \mathrm{~cm}$, the error is reduced twice and the vibration is reduced remarkably. 


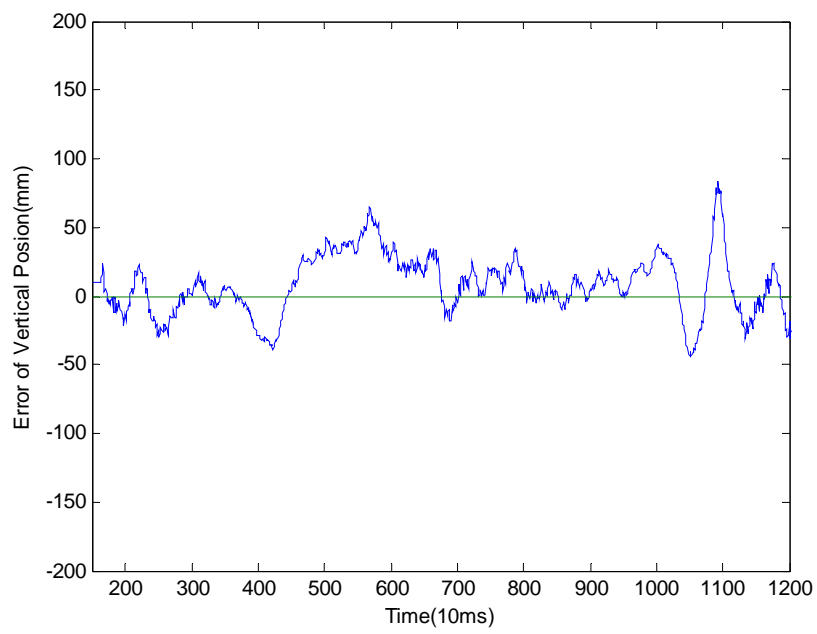

Figure 8: Error of vertical bucket's position with gravity compensation and gain scheduling

\section{CONCLUSIONS}

In this paper, we study the effect of the gravity compensation and gain scheduling. As the hydraulic system is different from electro motor system, it is difficult to control the system. So we use the feed forward of gain scheduling in the PI controller, we improve the performance of intelligent excavator system . We are planning to conduct a new research on contour control algorithm applied to the intelligent excavator.

\section{ACKNOWLEDGEMENT}

This research was supported by a grant(code:06 high technology fusion 001) from Construction Technology Innovation Program funded by Ministry of Construction \& Transportation of Korean government.

\section{REFERENCES}

Joseph, G. F.(2004), Development of a Haptic Backhoe Testbed, A Thesis of Master of Science, School of Mechanical Engineering, Georgia Institute of Technology, pp. 14-30.

Frimpong, S. and LI, Y.(2005), Virtual prototype simulation of hydraulic shovel kinematics for spatial characterization in surface mining operations, Int. J. of Surface Mining, Reclamation and Environment, Vol. 19, No. 4, pp. 238-250.

Adrian, B. and Peter I. C.(1999), A Pressure-Based Velocity Independent Friction Model for Asymmetric Hydraulic Cylinders, IEEE International Conference on Robotics \& Automation, Detroit.

Byung J. K. and Andrew E. Y.(1999), Nonlinear System Identification of Hydraulic Actuator Friction Dynamics Using A Hammerstein Model, IEEE International Conference on the Acoustics, Speech, and Signal Processing. 